\title{
Error analysis of an analog correlator for polarimetry
}

\author{
Jin-Taek Seong ${ }^{1 \mathrm{a})}$, Sung-Hyun Kim ${ }^{2 \mathrm{~b})}$, and Yong-Hoon $\mathrm{Kim}^{3 \mathrm{c})}$ \\ ${ }^{1}$ Department of Information and Communication Engineering, Honam University, \\ 417 Eodeung-daero, Gwangsan-gu, Gwangju, Republic of Korea \\ ${ }^{2}$ National Security Research Institute, \\ 1559 Yuseong-daero, Yuseong-gu, Daejeon, Republic of Korea \\ ${ }^{3}$ School of Mechnical Engineering, Gwangju Institute of Science and Technology, \\ 123 Cheomdangwagi-ro, Buk-gu, Gwangju, Republic of Korea \\ a)jtseong@honam.ac.kr \\ b)shkim@nsr.re.kr \\ c)yhkim@gist.ac.kr, Corresponding Author
}

\begin{abstract}
In this letter, we consider a performance evaluation of an analog correlator, and extract Stokes parameters from cross correlation between horizontal and vertical channels of electric fields for a fully polarimetric radiometer. To this end, we develop an analog correlator using $90^{\circ}$ and $180^{\circ}$ hybrid couplers and square-law detectors. In order for its performance analysis, we derive a closed form of a mathematical representation for Stokes parameters in terms of amplitude and phase responses of both channels, characteristics of hybrid couplers. As a result, the cross correlation of $U$ and $V$ of Stokes parameters is 0.92 and 0.96 , respectively. And the maximum return loss of the proposed analog correlator is less than $-23 \mathrm{~dB}$ for all directions. In addition, the peak-to-peak amplitude response of the proposed analog correlator is $1.9 \mathrm{~dB}$, and for the phase response as $9^{\circ}$. We compare numerical and measured results of $I$ and $U$ of Stokes parameters, and obtain their average errors as $0.4 \%$ and $14.9 \%$.
\end{abstract}

Keywords: analog correlator, polarimetric radiometer, stokes parameters Classification: Microwave and millimeter-wave devices, circuits, and modules

\section{References}

[1] F. T. Ulaby, et al:: Microwave Remote Sensing Active and Passive Volume I Microwave Remote Sensing Fundamentals and Radiometry (Artech House, Inc., 1981).

[2] N. Skou: Microwave Radiometer Systems: Design and Analysis (Artech House, Inc., 2006) 2nd ed.

[3] S. H. Yueh, et al:: "Polarimetric brightness temperatures of sea surfaces measured with aircraft K- and Ka-band radiometers," IEEE Trans. Geosci. Remote Sens. 35 (1997) 1177 (DOI: 10.1109/36.628785).

[4] C.-T. Li, et al.: “AMiBA wideband analog correlator,” Astrophys. J. 716 (2010) 746 (DOI: 10.1088/0004-637X/716/1/746).

[5] C. M. Holler, et al:: "A $2-20 \mathrm{GHz}$ analog lag correlator for radio interferometry,” IEEE Trans. Instrum. Meas. 61 (2012) 2253 (DOI: 10.1109/ 
TIM.2012.2184960).

[6] O. Koistinen, et al.: "Comparison of analog continuum correlators for remote sensing and radio astronomy," IEEE Trans. Instrum. Meas. 51 (2002) 227 (DOI: 10.1109/19.997817).

[7] R. C. Toonen, et al.: "An ultrawideband cross-correlation radiometer for mesoscopic experiments,” IEEE Trans. Instrum. Meas. 57 (2008) 2874 (DOI: 10.1109/TIM.2008.925011).

[8] C. Wang, et al.: "A compact analog complex cross-correlator for passive millimeter-wave imager,” IEEE Trans. Instrum. Meas. 66 (2017) 2997 (DOI: 10.1109/TIM.2017.2714501).

[9] Collett: Polarized Light: Fundamentals and Applications (Marcel Dekker Inc., New York, 1993).

[10] S.-H. Kim, et al.: "Characterization of material emissivity using 4-stokes W-band radiometer," IEEE Int. Geo. Remote Sensing Symposium (IGARSS) (2013) (DOI: 10.1109/IGARSS.2013.6723460).

\section{Introduction}

In the remote sensing, many works using polarimetric radiometers have obtained Stokes parameters which measures horizontal and vertical polarizations of electric fields $[1,2]$. The applicability of correlators is due to the linearly and circularly polarized thermal emission of objects, e.g., sea surfaces [3], cosmic background radiation [4]. For extracting Stokes parameters, both analog and digital approaches have been exploited to develop cross correlators [5]. While digital correlators have the benefits of controllable bandwidth and stability without cross coupling, analog correlators take the advantage of large bandwidth, high sensitivity, low cost, low power consumption, and tractable implementation $[6,7,8]$.

Fully polarimetric radiometers have a great effect on obtaining the Stokes parameters depending on the amplitude and phase errors between the vertical and horizontal electric fields [2]. In fact, for the measurement of the Stokes parameters in the ocean surface, the peak-to-peak of $Q$ and $U$ of the Stokes parameters is about 4-6 Kelvin, while for the $V$ parameter it is less [3]. In order to extract more accurate and sophisticated Stokes parameters, most of the studies of the polarimetric radiometers have been attempted to minimize their errors in the amplitude and phase responses. To this end, we propose a numerical approach for a performance analysis of a correlator which is one of the core components for the development of the fully polarimetric radiometer. The proposed approach can numerically evaluate the amplitude and phase responses of the correlator using input and output relations of components.

In this work, we develop an analog correlator with $2 \mathrm{GHz}$ of bandwidth using $90^{\circ}$ and $180^{\circ}$ hybrid couplers and square-law detectors for the extraction of the Stokes parameters in the fully polarimetric radiometer. As a result, the cross correlation of $U$ and $V$ of Stokes parameters is 0.92 and 0.96 , respectively. Also the maximum return loss of the proposed correlator is less than $-23 \mathrm{~dB}$ for all ports of directions. The peak-to-peak amplitude and phase responses of the proposed correlator is $1.9 \mathrm{~dB}$ and $9^{\circ}$. In order for performance analysis, we propose a closed form of a mathematical representation for the Stokes parameters with characteristics 
of the hybrid couplers. We evaluate the performance of the analog correlator with amplitude and phase differences between in-phase and out-phase directions for the hybrid couplers. And we compare performance results of the Stokes parameters between the numerical and measured outputs.

\section{Stokes parameters}

In this section, we define Stokes parameters in unites of brightness $\left(\mathrm{WHz}^{-1} \mathrm{~m}^{-2} \mathrm{sr}^{-1}\right)$. The radiation from an object is partly polarized, where the brightness temperatures for both horizontal and vertical polarizations are different with each other. In order to represent the polarization of these electric fields, a vector $\mathbf{S}$ of parameters called the Stokes parameters is defined as follows [9]:

$$
\mathbf{S}=\left[\begin{array}{c}
I \\
Q \\
U \\
V
\end{array}\right]=\frac{1}{z}\left[\begin{array}{c}
\left\langle V_{0}^{2}\right\rangle+\left\langle H_{0}^{2}\right\rangle \\
\left\langle V_{0}^{2}\right\rangle-\left\langle H_{0}^{2}\right\rangle \\
2 V_{0} H_{0} \cos \theta \\
2 V_{0} H_{0} \sin \theta
\end{array}\right],
$$

where $\langle\cdot\rangle$ denotes the time average, $H_{0}$ and $V_{0}$ are the horizontal and vertical signals with incident angle $\theta, z$ is the impedance of the medium propagating the electric field, $I$ describes the total intensity of the electric field, $Q$ represents the difference of the vertical and horizontal electric intensities, and $U$ and $V$ describe the real and imaginary parts of the cross correlation of the electric field. Note that $I^{2}=Q^{2}+U^{2}+V^{2}$ where $I, Q, U$, and $V$ are the Stokes parameters in units of antenna temperature $(\mathrm{K})$.

Taking Rayleigh-Jeans approximation, the Stokes vector $\mathbf{S}$ for the field in Eq. 1 can be rewritten as an equivalent brightness temperature $T_{B}(\mathrm{~K})[9]$ :

$$
T_{B}=\frac{\lambda^{2}}{k} \mathbf{S},
$$

where $\lambda$ is the wavelength and $k$ is Boltzmann's constant. In this work, we will analyze a newly developed polarimetric radiometer: $94 \mathrm{GHz}$ Fully Polarimetric Radiometer, see [10] for details. This instrument can measure all Stokes parameters by a cross correlation of horizontal and vertical polarization.

\section{Design analog correlator}

\subsection{Description of correlator}

Fig. 1 shows the structure of the analog correlator which consists of one phase discriminator, two hybrid couplers, and six square-law detectors. The first two hybrid couplers provide as power dividers for separating both intensities of the orthogonally polarized fields. The two inputs of the analog correlator are the vertically and horizontally polarized fields, i.e., $V_{0}$ and $H_{0}$, respectively. The phase discriminator has three $90^{\circ}$ and one $180^{\circ}$ hybrid couplers which play a role of cross correlation for the extraction of $U$ and $V$ Stokes parameters. The outputs of six hybrid couplers are directly fed into the inputs of six square-law detectors which convert powers of electric fields to voltages with low-pass filters. From subtraction and addition of the outputs of the detectors, we can acquire all Stokes parameters as the equivalent brightness temperature. 


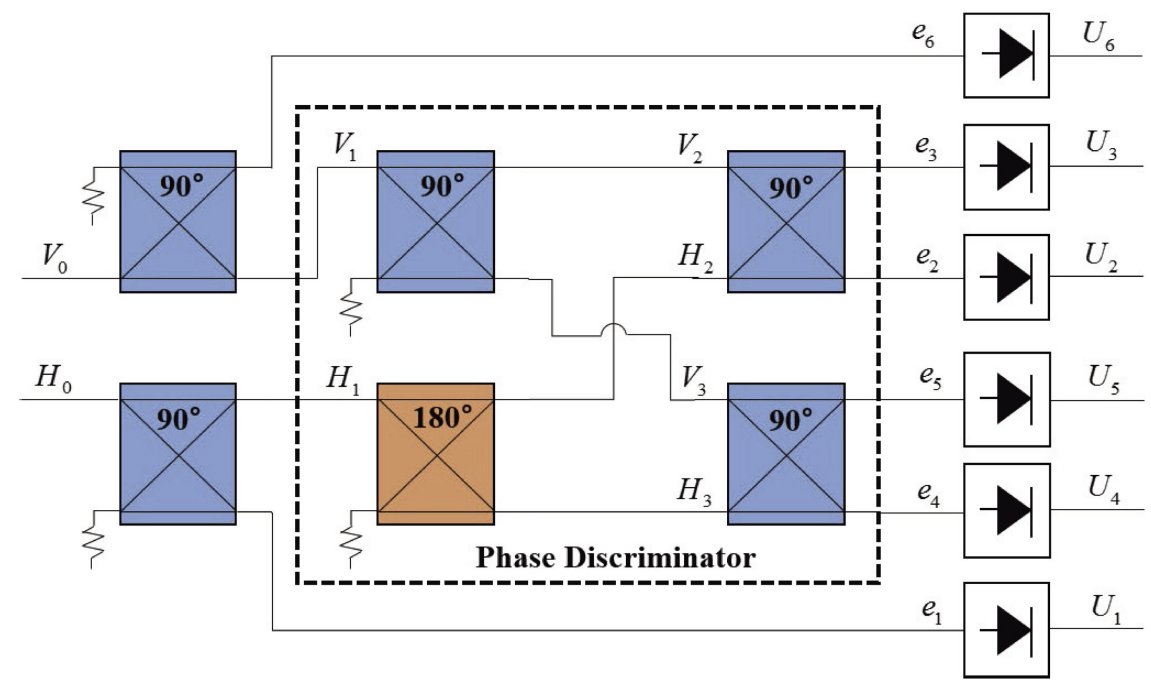

Fig. 1. Structure of the analog correlator.

Now we present the mathematical representation for the outputs of six squarelaw detectors, i.e., from $U_{1}$ to $U_{6}$ as shown in Fig. 1. For simplicity, we assume that the $90^{\circ}$ and $180^{\circ}$ hybrid couplers have the following characteristics. The five $90^{\circ}$ hybrid couplers have the same identical characteristics as coupling loss and phase shift between in-phase and out-phase directions. For the $90^{\circ}$ hybrid couplers, let $A_{1}$ and $A_{2}$ be the coupling loss, and $\alpha$ and $\alpha+\frac{\pi}{2}$ be the phase shift for the in-phase and out-phase directions, respectively. For the $180^{\circ}$ hybrid coupler, let $B_{1}$ and $B_{2}$ be the coupling loss, and $\beta$ and $\beta+\pi$ be the phase shift for the in-phase and out-phase directions. Suppose that two input signals of the correlator are $V_{0}$ and $H_{0} e^{-i \theta}$ for the vertical and horizontal channels. For this mathematical representation, we assume that effects of isolation and return loss for six hybrid couplers are ignored. Under these assumptions, we derive mathematical representation for the outputs of all the components along with the combination of the vertical and horizontal channels. For details, we describe as follows.

For instance, two outputs of the first two $90^{\circ}$ hybrid couplers can be written by

$$
V_{1}=V_{0} A_{1} \exp ^{-i \alpha}, \quad H_{1}=V_{0} A_{1} \exp ^{-i(\alpha+\theta)} .
$$

Using the same manner, other outputs of the hybrid couplers are given by

$$
\left\{\begin{array}{l}
e_{1}=H_{0} A_{2} \exp ^{-i\left(\alpha+\theta+\frac{\pi}{2}\right)}, \\
e_{2}=V_{0} A_{1}^{2} A_{2} \exp ^{-i\left(3 \alpha+\frac{\pi}{2}\right)}+H_{0} A_{1}^{2} B_{1} \exp ^{-i(2 \alpha+\beta+\theta)}, \\
e_{3}=V_{0} A_{1}^{3} \exp ^{-i 3 \alpha}+H_{0} A_{1} A_{2} B_{1} \exp ^{-i\left(2 \alpha+\beta+\theta+\frac{\pi}{2}\right)}, \\
e_{4}=V_{0} A_{1} A_{2}^{2} \exp ^{-i(3 \alpha+\pi)}+H_{0} A_{1}^{2} B_{2} \exp ^{-i(2 \alpha+\beta+\theta+\pi)}, \\
e_{5}=V_{0} A_{1}^{2} A_{2} \exp ^{-i\left(3 \alpha+\frac{\pi}{2}\right)}+H_{0} A_{1} A_{2} B_{2} \exp ^{-i\left(2 \alpha+\beta+\theta+\frac{3 \pi}{2}\right)}, \\
e_{6}=V_{0} A_{2} \exp ^{-i\left(\alpha+\frac{\pi}{2}\right)} .
\end{array}\right.
$$

By taking the average of all the outputs of the hybrid couplers for the detection, we can obtain $U_{1}$ to $U_{6}$, and then represent a form of the following matrix as 


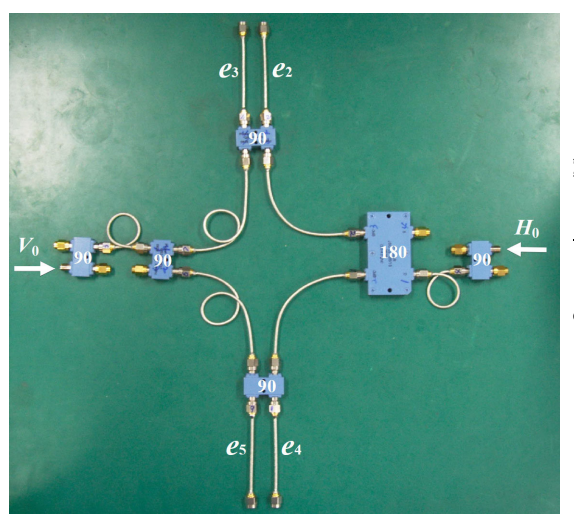

(a)

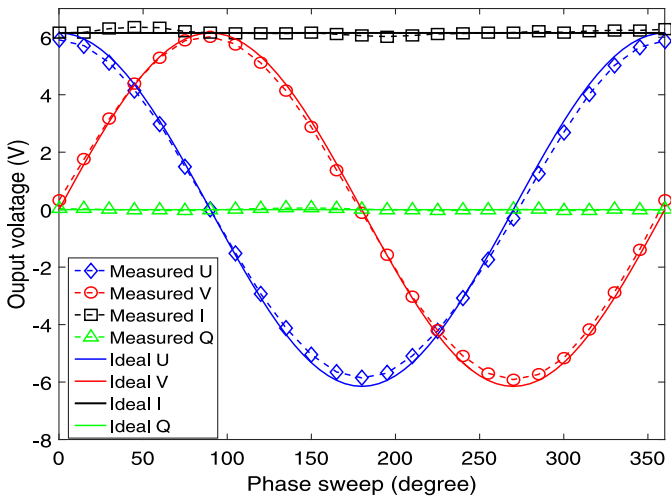

(b)

Fig. 2. (a) Implementation of the proposed analog correlator with $90^{\circ}$ and $180^{\circ}$ hybrid couplers. (b) Output voltages of $I, Q, U$, and $V$ of the Stokes parameters at $3 \mathrm{GHz}$ with measured (dots lines) and ideal (solid lines) results.

$\left[\begin{array}{l}U_{1} \\ U_{2} \\ U_{3} \\ U_{4} \\ U_{5} \\ U_{6}\end{array}\right]=\left[\begin{array}{cccc}0 & A_{2}^{2} & 0 & 0 \\ A_{1}^{4} A_{2}^{2} & A_{1}^{4} B_{1}^{2} & 2 A_{1}^{4} A_{2} B_{1} \sin (\beta-\alpha) & 2 A_{1}^{4} A_{2} B_{1} \cos (\beta-\alpha) \\ A_{1}^{6} & A_{1}^{2} A_{2}^{2} B_{1}^{2} & -2 A_{1}^{4} A_{2} B_{1} \sin (\beta-\alpha) & -2 A_{1}^{4} A_{2} B_{1} \cos (\beta-\alpha) \\ A_{1}^{2} A_{2}^{4} & A_{1}^{4} B_{2}^{2} & 2 A_{1}^{3} A_{2}^{2} B_{2} \cos (\beta-\alpha) & 2 A_{1}^{3} A_{2}^{2} B_{2} \sin (\beta-\alpha) \\ A_{1}^{4} A_{2}^{2} & A_{1}^{2} A_{2}^{2} B_{2}^{2} & -2 A_{1}^{3} A_{2}^{2} B_{2} \cos (\beta-\alpha) & -2 A_{1}^{3} A_{2}^{2} B_{2} \sin (\beta-\alpha) \\ A_{2}^{2} & 0 & 0 & 0\end{array}\right]\left[\begin{array}{c}V_{0}^{2} \\ H_{0}^{2} \\ V_{0} H_{0} \cos \theta \\ V_{0} H_{0} \sin \theta\end{array}\right]$.

For the ideal correlator, the coefficients of the hybrid couplers are $A_{1}=A_{2}=$ $B_{1}=B_{2}=1 / \sqrt{2}$ and $\alpha=\beta=0$. And Stokes parameters are obtained from $I=$ $U_{6}+U_{1}, Q=U_{6}-U_{1}, U=U_{4}-U_{5}$ and $V=U_{2}-U_{3}$.

\subsection{Performance analysis of analog correlator}

In this section, we analyze and compare the performance of the analog correlator. As shown in Fig. 2(a), we develop the wideband analog correlator with approximate $2 \mathrm{GHz}$ of the effective bandwidth, i.e., 2 to $4 \mathrm{GHz}$. We used commercial $90^{\circ}$ and $180^{\circ}$ hybrid couplers provided by PULSAR microwave co. Ltd. For the phase balance, we compensate phase difference of both $90^{\circ}$ and $180^{\circ}$ hybrid couplers by using coaxial cables. All the square-law detectors having under $10 \mathrm{~dB}$ of return loss over 2 to $4 \mathrm{GHz}$ are designed by Schottky diodes (HSCH9161) and precise amplifiers (AD620). Identically characteristic six square-law detectors are mounted on the analog correlator.

Fig. 2(b) shows that the output voltages for all the Stokes parameters with the phase sweep from 0 to $360^{\circ}$ are measured. Comparison with the ideal Stokes parameters, this observation shows that the correlation coefficients of the $U$ and $V$ Stokes parameters are 0.92 and 0.96 , respectively. This means that the cross correlation between the vertical and horizontal channels properly occurs.

Table I shows the results of other state of the arts including our work in order to compare the performance of the analog correlator. The analog correlators are mainly used to obtain vertical and horizontal polarizations of electromagnetic 


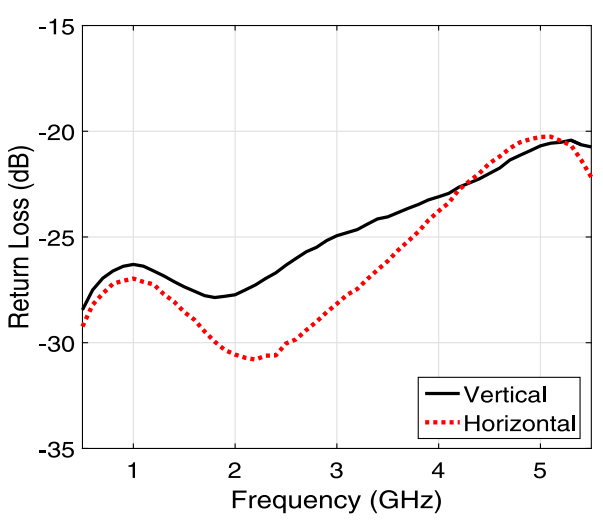

(a)

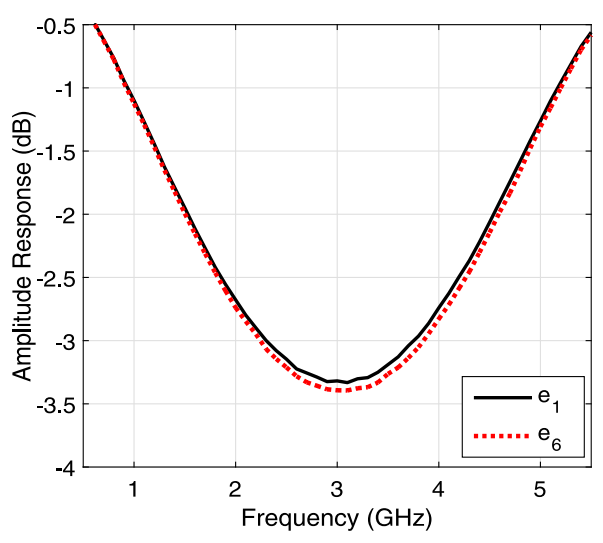

(b)

Fig. 3. (a) Return loss of the vertical (solid line) and horizontal (dots line) channels, and (b) Amplitude response of the output $e_{1}$ (solid line) and $e_{6}$ (dots line) in the analog correlator.

waves in astronomy and radiometer. The maximum return loss of the analog correlator is less than $-23 \mathrm{~dB}$ for the vertical and horizontal channels as shown in Fig. 3(a). And as shown in Fig. 3(b) and 4, the maximum peak-to-peak amplitude and phase responses of the proposed analog correlator obtained from this work are $1.9 \mathrm{~dB}$ and $9^{\circ}$, respectively. Recently, the studies of development of radiometer systems have been efforts to improve sensitivity by increasing bandwidth due to high frequencies, i.e., terahertz. Compared to other studies $[5,6,7,8]$, we achieve highly sensitive performance of the proposed analog correlator as shown in Table I.

Next we compare the performance of the analog correlator with respect to the amplitude and phase error responses using Eq. 5. Let $\epsilon$ and $\delta$ be the amplitude and phase differences between the in-phase and out-phase directions for the $90^{\circ}$ and $180^{\circ}$ hybrid couplers as $\epsilon=A_{1}-A_{2}=B_{1}-B_{2}, \delta=\beta-\alpha$. We define the following coefficients of the errors for Stokes parameters as $\Delta I=\left|\frac{I-I_{0}}{I}\right|, \Delta Q=\left|\frac{Q-Q_{0}}{Q}\right|$,

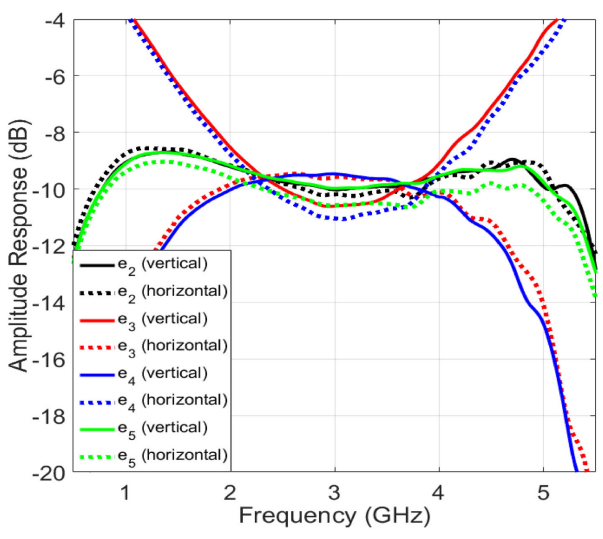

(a)

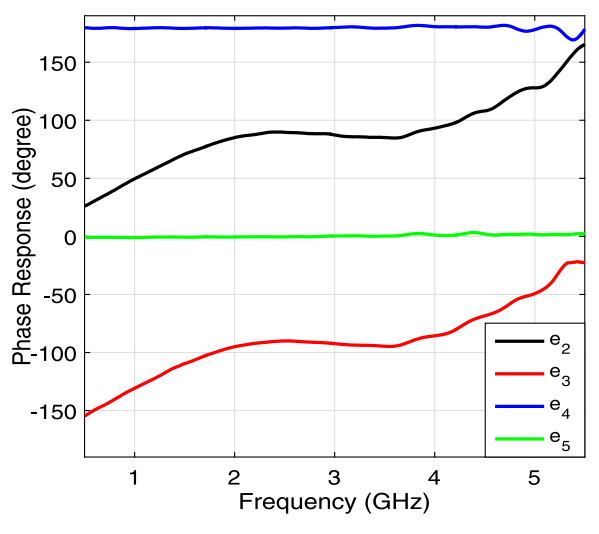

(b)

Fig. 4. (a) Amplitude response of the output $e_{2}$ (black), $e_{3}$ (red), $e_{4}$ (blue), and $e_{5}$ (green) for the vertical and horizontal channels, (b) Phase response of the output $e_{2}$ (black), $e_{3}$ (red), $e_{4}$ (blue), and $e_{5}$ (green) for the phase difference between the vertical and horizontal channels. 
Table I. Summary of the state of the arts for analog correlators.

\begin{tabular}{|c|c|c|c|c|c|c|c|}
\hline Reference & Architecture & $\begin{array}{c}\text { Bandwidth } \\
(\mathrm{GHz})\end{array}$ & Correlation & $\begin{array}{c}\text { Max. } \\
\text { return loss } \\
(\mathrm{dB})\end{array}$ & $\begin{array}{c}\text { Peak-peak } \\
\text { amplitude } \\
(\mathrm{dB})\end{array}$ & $\begin{array}{c}\text { Peak-peak } \\
\text { phase } \\
(\text { degree })\end{array}$ & Application \\
\hline Our work & Power divider & $2-4$ & $>0.92$ & $<-23$ & 1.9 & 9 & Radiometer \\
\hline$[5]$ & Power divider & $1.25 /$ channel & - & $<-6$ & 2.0 & - & Astronomy \\
\hline$[6]$ & Multiplier & $0.09-0.52$ & - & $<-17$ & 3.9 & 4 & Radiometer \\
\hline$[6]$ & Mixer & $0.1-1.0$ & - & $<-19$ & $<1$ & 17 & Astronomy \\
\hline$[7]$ & Delay line & $5.08-14.78$ & $>0.95$ & - & - & - & Radiometer \\
\hline$[8]$ & Power divider & $1.5-2.5$ & $>0.96$ & $<-8$ & $<1.5$ & $<11$ & Radiometer \\
\hline
\end{tabular}

$\Delta U=\left|\frac{U-U_{0}}{U}\right|$ and $\Delta V=\left|\frac{V-V_{0}}{V}\right|$ where $I_{0}, Q_{0}, U_{0}$ and $V_{0}$ denote the values of Stokes parameters in the ideal case. In Fig. 5, we show how varying the amplitude and phase differences affect the performance of Stokes parameters. While the amplitude difference $\epsilon$ of the hybrid coupler affects to $I, U$ and $V$ Stokes parameters, $Q$ Stokes parameters does not change due to assumption that all $90^{\circ}$ hybrid couplers are the same characteristics. Because $I$ and $Q$ Stokes parameters are not dependent of the phase difference, the errors of only $U$ and $V$ Stokes parameters in the phase difference occur as shown in Fig. 5(b) in which $U$ and $V$ are orthogonal with each other.

In order for comparison of the performance between the numerical and measured results for Stokes parameters, we evaluate the results of $\Delta I$ and $\Delta U$ as shown in Fig. 6. For $\Delta I$, the numerical and measured results are well matched, then its average error is $0.4 \%$. However, due to the non-ideal characteristics of all the $90^{\circ}$ and $180^{\circ}$ hybrid couplers, the measured cross correlation of the horizontal and vertical channels are different from the numerical approach in which the average error of $\Delta U$ is $14.9 \%$.

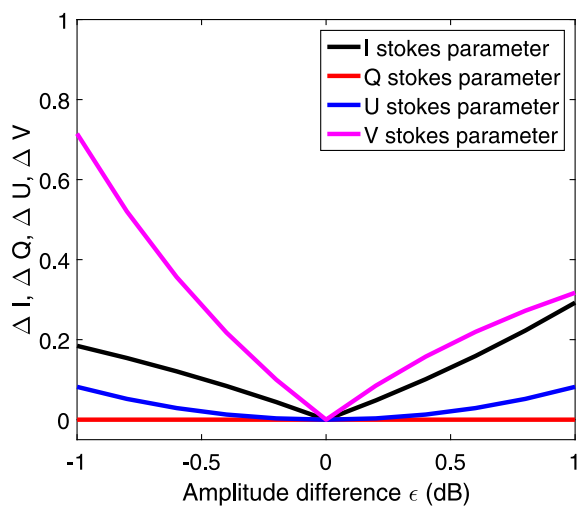

(a)

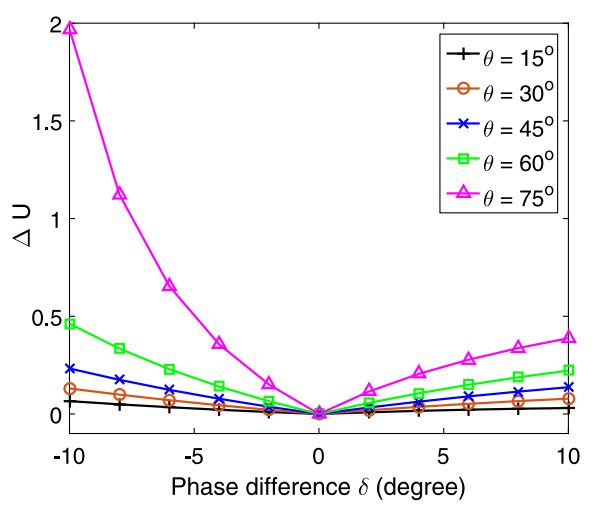

(b)

Fig. 5. Errors of Stokes parameters with: (a) amplitude difference $\epsilon$ for $I, Q, U$, and $V$, (b) phase difference $\delta$ of the hybrid coupler for $U$. 


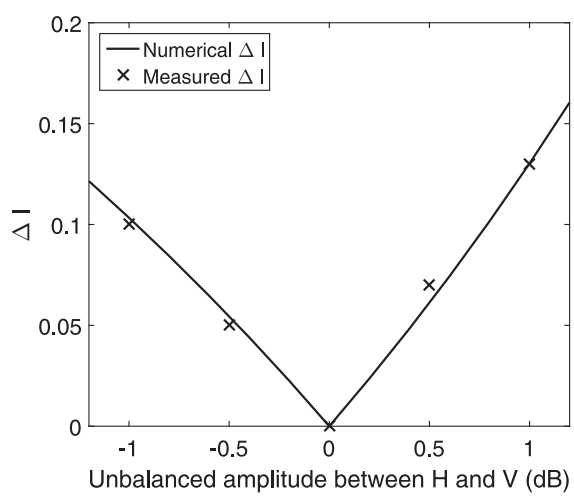

(a)

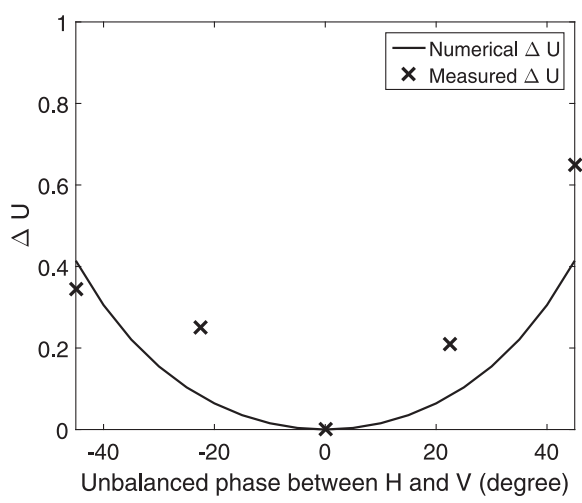

(b)

Fig. 6. Comparison of the performance with the numerical (solid lines) and measured (marked) errors for: (a) $\Delta I$, (b) $\Delta U$.

\section{Conclusion}

We evaluated the performance of the analog correlator for the fully polarimetric radiometer, and extracted Stokes parameters from the cross correlation between the horizontal and vertical channels of electric fields. We designed the analog correlator using $90^{\circ}$ and $180^{\circ}$ hybrid couplers and square-law detectors. We derived the mathematical representation for Stokes parameters with respect to the characteristics of device components. We not only evaluated the effects on errors of Stokes parameters, but also compared the numerical and measured results in terms of amplitude and phase responses of both channels.

\section{Acknowledgments}

This study was supported by research fund from Honam University, 2017, and by the National Research Foundation of Korea (NRF) grant funded by the korean government (NRF-2017R1C1B5075823). 